\title{
Collapse of granular media subjected to wetting
}

\author{
Fatima Zahra El Korchi ${ }^{1, *}$, Frédéric Jamin ${ }^{1,2}$, and Moulay Saïd El Youssoufi ${ }^{1,2}$ \\ ${ }^{1}$ LMGC, Univ. Montpellier, CNRS, Montpellier, France. \\ ${ }^{2}$ MIST Lab., IRSN, CNRS, Univ. Montpellier, France.
}

\begin{abstract}
This paper focuses on the collapse of granular materials subjected to wetting action. For soils, the collapse potential depends on several parameters such as liquid limit, matric suction, compactness, initial water content and the amount of fine particles. The effect of grain size, which plays a key role in the rearrangement of grains, remains little studied and poorly understood. To investigate the capillary origin of the collapse phenomenon, we present an experimental study on macroscopic and local scales. Our results show the effect of grain size and water content on collapse.
\end{abstract}

\section{Introduction}

In the pendular regime, the presence of isolated capillary bridges in a granular medium generates a cohesive force by capillary action between grains. These forces are responsible for the stability of a sand castle for example. The capillary forces depend on the quantity of liquid contained in the medium [11].

During wetting, the capillary bridges merge because the local geometry of the grains no longer allows the formation of isolated bridges. Thereby, big changes in the morphology liquid must take place in the granular assemblies, which must necessarily change its behavior. This phenomenon is still little studied experimentally considering the complexity of the phenomenon of coalescence at local scale. Deposited solutes and/or capillary bridges can bond the solid particles in soils. When these soils are subjected to wetting action, they can undergo a collapse phenomenon.

The collapse potential of soils depends on several parameters such as liquid limit [8,2], suction [12], compactness $[12,4]$, initial water content $[12,4]$ and the amount of fine particles $[3,1]$. The effect of grain size, which plays a key role in the rearrangement of grains, remains little studied and poorly understood.

This communication will focus on the collapse of granular media subjected to wetting action. To investigate the origin of capillary collapse phenomenon, we present an experimental study in macroscopic and local scales. Glass beads form the used samples. The wicking tests are performed on the macroscopic scale of a VER sample and at the local scale of 3 and 4 grains.

\section{Macroscopic scale study}

\subsection{Materials and experimental protocol Materials}

\footnotetext{
* Corresponding author: fatima-zahra.el-korchi@umontpellier.fr
}

\subsubsection{Materials}

The used samples are made by glass beads of grain size $\Phi:<50 \mu \mathrm{m}, 60-100 \mu \mathrm{m}, 80-200 \mu \mathrm{m}$ and $300-500$ $\mu \mathrm{m}$. Some of the used material characteristics are presented in Table 1.

Table 1. Physical properties of the different samples.

\begin{tabular}{|c|c|c|c|}
\hline Materials & $\begin{array}{c}\text { Grain sizes } \\
\Phi \\
(\boldsymbol{\mu m})\end{array}$ & $\begin{array}{c}\text { Specific } \\
\text { density } \\
\boldsymbol{\rho}_{\mathbf{s}} \\
\left(\mathbf{k g} / \mathbf{m}^{3}\right)\end{array}$ & $\begin{array}{c}\text { Liquid limit } \\
\boldsymbol{w}_{\boldsymbol{L}} \\
(\mathbf{\%})\end{array}$ \\
\hline \multirow{3}{*}{ Glass beads } & $<50$ & 2450 & 23 \\
\cline { 2 - 4 } & $60-100$ & 2470 & 25 \\
\cline { 2 - 4 } & $80-200$ & 2480 & 24 \\
\cline { 2 - 4 } & $300-500$ & 2500 & - \\
\hline
\end{tabular}

Fig. 1 shows the retention curves for all grain sizes obtained by the filter paper technique (Whatman "42" paper). A modelling of the retention curves is also plotted in Fig. 1 based on Fredlund and Xing [6].

\subsubsection{Experimental protocol}

The samples are cylindrical in shape with an initial height $H_{0}=94 \mathrm{~mm}$ and an initial diameter $D_{0}=50 \mathrm{~mm}$. The samples were prepared with an initial compactness $c_{0}=0.59$ and initial water contents $w_{0}=5 \%$. The initial water content $w_{0}$ allows the creation of the capillary bridges in the sample that are at the origin of their mechanical strength by cohesion. The principle involves applying low water pressure $\left(u_{w}=5 \mathrm{kPa}\right)$ at the bottom of the sample by the Pressure - Volume Controller while maintaining atmospheric air pressure at the top of the sample $\left(u_{a}=0 \mathrm{kPa}\right)$ as explained in El Korchi et al. [5]. During the test, the water content $w$ within the sample was determined by the volume of injected water $V_{w}$, 
directly reported by the Pressure - Volume Controller at a given time $t$ of the wetting path [5].

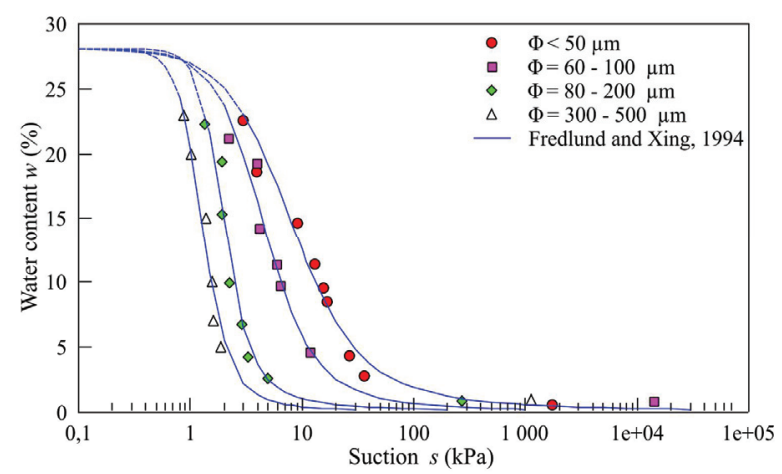

Fig. 1. Retention curves for different glass beads $(c=0.59)$.

\subsection{Experimental results}

\subsubsection{Highlighting of collapse}

The wetting test results obtained on glass beads of grain size distributions $\Phi<50 \mu \mathrm{m}$, with compactness $c_{0}=$ 0.59 and initial water content $w_{0}=5 \%$, are shown in Fig. 2.

During the ascent of water in the granular sample, axial and radial deformations $\varepsilon_{a}$ and $\varepsilon_{r}$ evolve. A positive axial deformation corresponds to a decrease of the height of the sample and a negative radial deformation corresponds to an increase of its diameter. For a critical water content, deformations undergo a sudden variation assigned to the collapse phenomenon. This critical water content is subsequently called collapsing water content and noted $w_{\text {coll }}$ in Fig. 2.

In what follows, we define the final axial deformation $\varepsilon_{a}^{f}$ as the maximum axial deformation due to the wetting while the amplitude of collapse $A$ is defined as the axial deformation due to the sudden collapse of the sample as shown in Fig. 2.

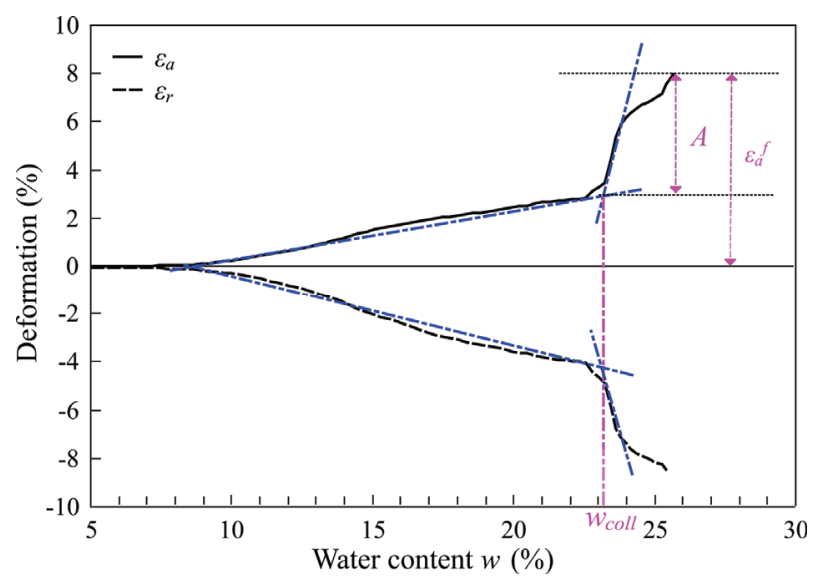

Fig. 2. Evolution of axial $\varepsilon_{a}$ and radial $\varepsilon_{r}$ deformations during wetting for glass beads $\Phi<50 \mu \mathrm{m}\left(w_{0}=5 \%\right.$ and $\left.\mathrm{c}_{0}=0.59\right)$.

\subsubsection{Effect of grain size}

The effect of grain size on deformations $A$ and $\varepsilon_{a}^{f}$ and collapsing water content $w_{\text {coll }}$ is shown in Fig. 3. These figures are plotted with a horizontal uncertainty bar showing the spreading of the grain size of the glass beads.

Axial deformations $\varepsilon_{a}^{f}$ and $A$ decreased nonlinearly with the grain size (Fig. 3.a). This result shows the high sensitivity of small granularity samples to wetting. This sensitivity can be explained by the different microstructural morphology of the samples, having the same volume fraction. The finer the grain size, the more dominant are the capillary forces relative to the gravity [9].

The collapsing water content appears to depend on the grain size. A slight increase in the water content with the grain size shows the sensitivity of the granular materials for the wetting (Fig. 3.b).
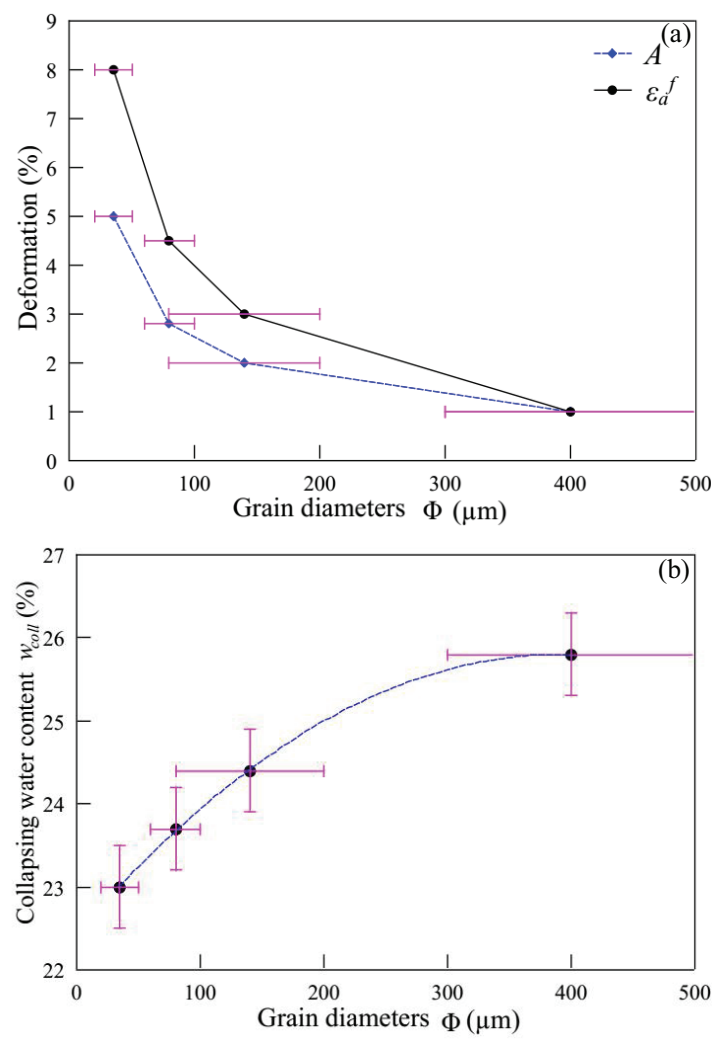

Fig. 3. a. axial deformation $\left(\varepsilon_{a}^{f}\right.$ and $A$ ) and b. collapsing water content $w_{\text {coll }}$ as a function of grain size.

\section{Local scale study}

\subsection{Materials and experimental protocol}

\subsubsection{Materials}

At local scale, wetting tests were conducted on three and four borosilicate glass beads grade 25 and $8 \mathrm{~mm}$ in diameter. The capillary force is measured by the apparatus shown in Fig. 4. The used experimental device is composed of a precision balance 1/1000, a micrometric table on which is fixed an upper glass bead (Fig. 4.b) as well as supports of two or three spheres 
(Fig. 5.a and Fig. 5.c) respectively to form 3 and 4 spheres configurations. Distilled water is used to form the capillary bridges between the glass beads. The provision of water in bridges is done with micro-syringes with the accuracy of $0.1 \mu \mathrm{l}$ and the volume of 5 and 10 $\mu 1$.

\subsubsection{Experimental protocol}

For the three sphere configuration, the centers of the three glass beads form a triangle in the vertical plane as shown in Fig. 5.a. and for the four sphere configuration, the centers of spheres form a tetrahedron (Fig. 5.b).

The upper sphere is fixed on the micrometer table which allows applying distances, denoted $D_{l}$ between the centers of the upper and the lower spheres. The lower spheres are fixed on a support with a distance, denoted $D_{2}$, separating their centers.

The test consists in forming two or three bridges between the upper sphere and the two or three lower spheres. Then, water is injected in each bridge with the micro-syringe (no bridges are formed between the lower spheres). For each water volume injected $V_{w}$ into the bridges, the axial capillary force $F_{c a p}$ is obtained with the expression 1 [7]:

$$
F_{\text {cap }}=\left(m_{s}+m_{w}-m_{a}\right) \cdot g
$$

where $m_{s}$ is the mass of the support with the fixed spheres, $m_{w}=\rho_{w} V_{w}$ is the water mass cumulated, $m_{a}$ is the apparent mass measured for each water volume injected, and $g$ the acceleration of gravity.
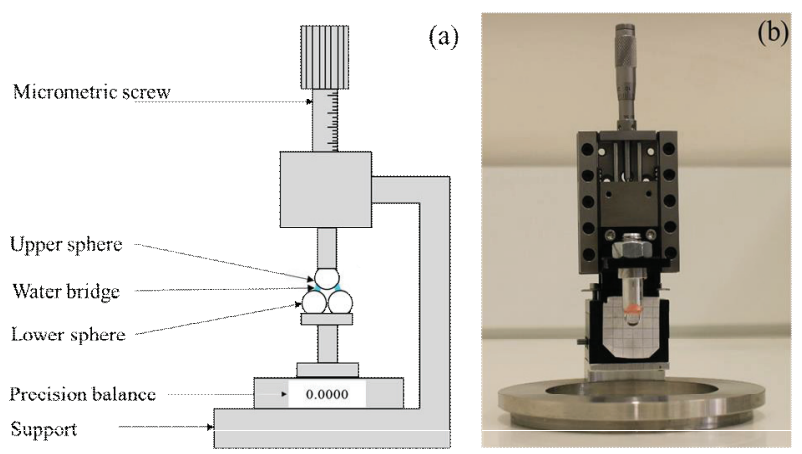

Fig. 4. a. Schematic of the used apparatus at a local scale, b. the micrometer table.
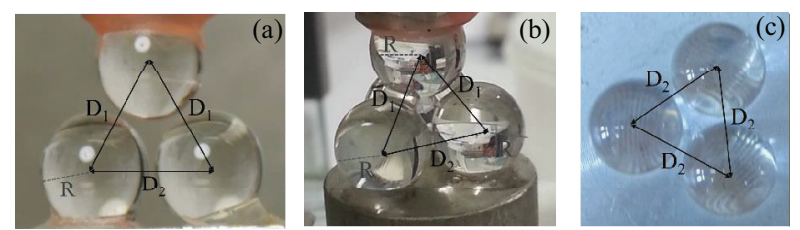

Fig. 5. a. Image of the three spheres configuration a. and the four spheres configuration $\mathrm{b}$. with distances $D_{l}$ and $D_{2}$, and c. image of the three spheres with the intergranular distances $D_{2}$.

\subsection{Experimental results}

\subsubsection{Three spheres configuration}

Fig. 6 shows images of the three sphere configuration for each cumulative water volume $V_{w}\left(D_{1}=8.7 \mathrm{~mm}\right.$ and $D_{2}$ $=8.3 \mathrm{~mm})$. After forming the two capillary bridges $2 \mu \mathrm{l}$ each $\left(V_{w}=4 \mu \mathrm{l}\right)$, adding $2 \mu \mathrm{l}$ of water in each bridge increases their radii. When the two bridges touch, the coalescence phenomenon occurs to form a single bridge between the 3 spheres $\left(V_{w}=20 \mu \mathrm{l}\right)$. By increasing the volume of water, a fused bridge lies between the 3 spheres $\left(V_{w}=24,28,32,36,40\right.$ and $\left.44 \mu 1\right)$. We begin to see the effect of gravity on the coalesced bridge, by exceeding a volume of water $V_{w}=36 \mu \mathrm{l}$.

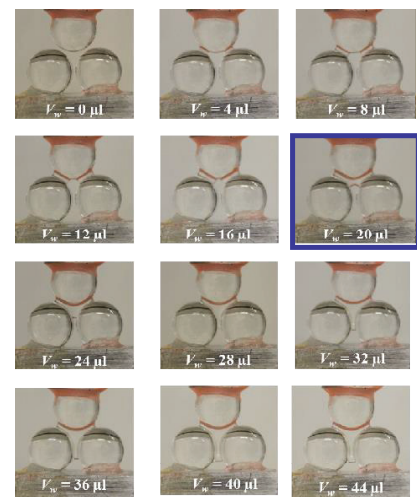

Fig. 6. Photos of three spheres configuration with different cumulative water volume $V_{w}\left(D_{l}=8,7 \mathrm{~mm}\right.$ et $\left.D_{2}=8,3 \mathrm{~mm}\right)$.

For the same intergranular distances, Fig. 7.a presents the capillary force depending on the volume of water injected $V_{w}$. This figure shows four different domains. In the first, corresponding to the water volume between 0 and $4 \mu 1$, the capillary force greatly increases from 0 to $6.10^{-4} \mathrm{~N}$ following the creation of bridges between spheres. The second, corresponding to the volume of water of between 4 to $16 \mu \mathrm{l}$, where there is a stabilization of the capillary force with the volume of water injected into the two separate bridges. In the third, during the merger, the capillary force increases sharply (Fig. $\left.6\left(V_{w}=20 \mu \mathrm{l}\right)\right)$. The peak of the capillary force $F_{c a p}$ corresponds to the merger of two bridges and the development of the bridge between the 3 grains (Fig. 6 $\left.\left(V_{w}=24 \mu \mathrm{l}\right)\right)$. And the fourth, following the merger of the two bridges corresponds to water volumes of $24 \mu 1$ to $36 \mu 1$, where the capillary force decreases slightly with the increasing volume of the coalesced bridge.

\subsubsection{Four spheres configuration}

For intergranular distances $D_{1}=8.4 \mathrm{~mm}$ and $D_{2}=8.3$ $\mathrm{mm}$, Fig. 7.b shows the capillary force as a function of volume of water $V_{w}$. As in the case of the three sphere configuration, changes in capillary force has(a)four domains in which the trend is different. The merger of the bridges is done in 3 steps, the first corresponds to the volume $V_{w}$ of $30 \mu \mathrm{l}$ where the two adjacent first bridges merge. When adding a water volume of $4 \mu \mathrm{l}$, the merger is made between the first coalesced bridge and the third bridge. And finally, with the addition of $4 \mu 1$, fusion is done on the 3 faces of the tetrahedron. At this stage, the capillary force is maximum $18 \cdot 10^{-4} \mathrm{~N}\left(V_{w}=38 \mu \mathrm{l}\right)$. 

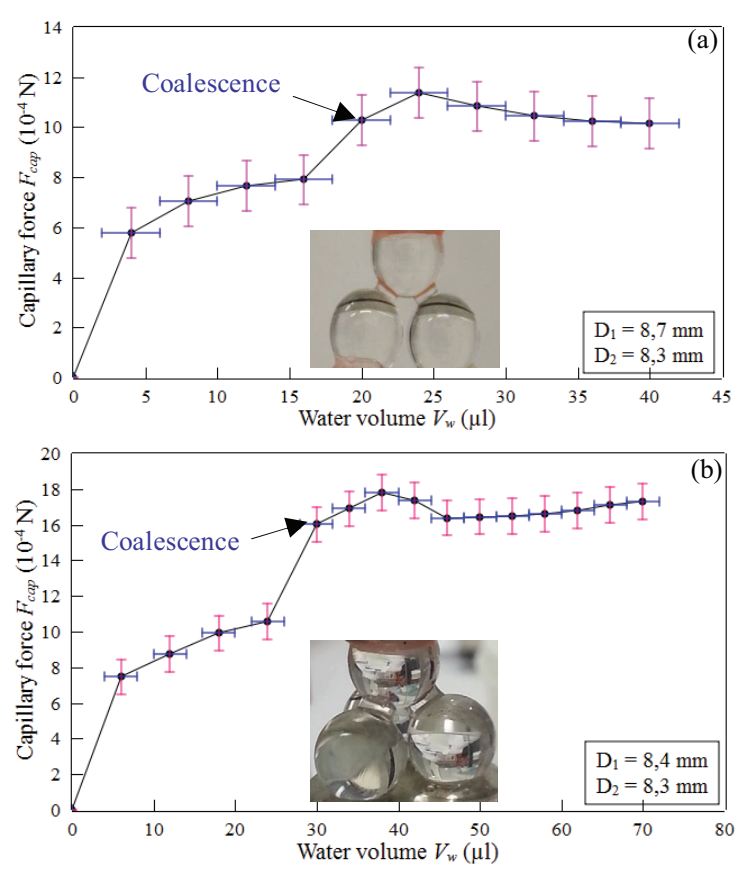

Fig. 7. Evolution of capillary force as function of water volume for a. 3 spheres and b. 4 spheres.

\section{Discussion and conclusion}

Wetting in a granular material induced volume changes of a cylindrical sample (Fig. 2). The deformations due to wetting took an abrupt form for a critical water content $w_{\text {coll }}$, due to which the sample instantly lost its mechanical strength. These abrupt deformations correspond to a homogeneous water content in the sample, contrary to the progressive deformations [5]. The observed collapse seems to result from coalescence of the capillary bridges between glass beads.

The increase of the water content, during wetting, impacts the finer grain sample more. This effect is illustrated on Fig. 3.a. We can notice that the fine grains present higher axial deformation $\left(A\right.$ and $\varepsilon_{a}^{f}$ ). Based on the retention curve (Fig. 1), changes in the suction $\Delta s$, between the initial state $\left(w=w_{0}\right)$ and collapse $w=w_{\text {coll }}$, are shown as function of grain size in Fig. 8. It shows the same trend as the axial deformations $\left(A\right.$ and $\left.\varepsilon_{a}^{f}\right)$, which explains the effect of suction on the collapse.

At local scale, the capillary force increases due to the formation of capillary bridges (Fig. 7). This domain corresponds to low water contents when the capillary cohesion increases with the water content as shown by Soulié et al. [11] and Richefeu et al. [10]. During wetting, the capillary bridges merge and the liquid phase becomes continuous in the sample. In this state, the capillary force suddenly increases by scoring a jump depending on the volume of water. After bridge fusion, the capillary force decreases slightly (Fig. 7) with higher water volume and seems to be the origin of the observed collapse.

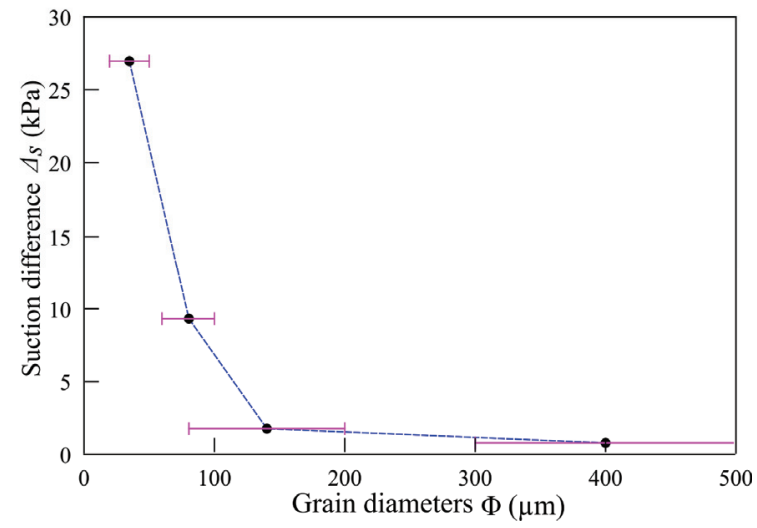

Fig. 8. Evolution of suction difference as a function of grain size.

\section{References}

1. T. Ayadat, B. Belouahri, R. Ait Ammar, RFG, 83, 73-83 (1998)

2. T. Ayadat, S. Ouali, RFG, 86, 53-56 (1999)

3. L. Barden, A. McGow, K. Collins, Engineering Geology, 7, 49-60 (1973)

4. P. Delage, Y. J. Cui, P. Antoine, Proceedings of International Conference on Problematic Soils, $517-$ 540 (2005)

5. F. Z. El Korchi, F. Jamin, M. El Omari, M. S. El Youssoufi, EJECE, DOI: 10.1080/19648189.2016.1177602 (2016)

6. D. G. Fredlund, A. Xing, Rev. Can. Géotech., 31, 521-532 (1994)

7. J. P. Gras, Thèse de doctorat, Montpellier (2011)

8. H. Gibbs, J. Bara, Special Technical Publication $\mathrm{N}^{\circ}$ 322, ASTM, 277-283 (1962)

9. J. K. Mitchell, Wiley Inter Science, Second edition (1993)

10. V. Richefeu, M. S. El Youssoufi, F. Radjaï, Phys. Rev., 73: 05130 (2006)

11. F. Soulié, M.S. El Youssoufi, F. Cherblanc, C. Saix, EPJ, 21, 349-357 (2006)

12. R. Tadepalli, H. Rahardjo, D. G. Fredlund, GTJ, 15, 115-122 (1992) 